\title{
Time-dependent Hartree-Fock studies of superheavy molecules
}

\author{
M. R. Strayer \\ Department of Physics and Wright Nuclear Structure Laboratory, Yale University, New Haven, Connecticut 06520 \\ R. Y. Cusson \\ Department of Physics, Duke University, Durham, North Carolina 27706 \\ H. Stoecker* \\ Gesellschaft fur Schwerionenforschung, D-6100 Darmstadt, Federal Republic of Germany \\ J. A. Maruhn and W. Greiner \\ Institut fur Theoretische Physik der Universität Frankfurt, D-6000 Frankfurt/Main, Federal Republic of Germany
}

(Received 6 December 1982)

\begin{abstract}
The time dependent Hartree-Fock approximation is used to study the dynamical formation of long-lived superheavy nuclear complexes. The effects of long-range Coulomb polarization are treated in terms of a classical quadrupole polarization model. Our calculations show the existence of "resonantlike" structures over a narrow range of bombarding energies near the Coulomb barrier. Calculations of ${ }^{238} \mathrm{U}+{ }^{238} \mathrm{U}$ are presented and the consequences of these results for supercritical positron emission are discussed.
\end{abstract}

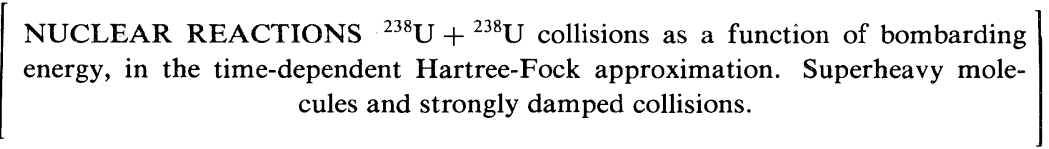

\section{INTRODUCTION}

The possible existence and subsequent properties and decay modes of long lived superheavy nuclei have been actively pursued for the past 15 years. ${ }^{1}$ Experimental searches $^{2}$ presupposing their natural occurrence in small abundances have proved unsuccessful, and most of the current work centers on accelerator based synthesis using heavy ion fusion and strongly damped reactions. ${ }^{3}$ The reactions are usually carried out at relatively high energies and place limits on the production cross sections ranging from $10^{-28} \mathrm{~cm}^{2}$ to $10^{-36} \mathrm{~cm}^{2}$ depending on the details of each particular reaction and on estimates for the decay time of the superheavy species. ${ }^{4}$ Most of this work assumes the existence of stable regions in mass and charge arising through the doubly magic shell closure near proton and neutron number ${ }^{5-7} Z=114$ and $N=184$. Greiner ${ }^{8}$ has also suggested that a superheavy nuclear quasimolecule might possibly be formed during low energy heavy ion collisions. In contrast to superheavy nuclei, these complexes have a separate two-center shell structure similar to the projectile and target fragments rather than that corresponding to the compound nucleus. ${ }^{9}$ Thus the formation and decay properties for these structures are more closely related to the dynamics of the reaction rather than the properties of the compound system. As such, these structures represent possible probes of heavy ion reactions.

The formation of heavy nuclear complexes using very low energy heavy ion reactions has been studied by Armbruster et al. Evaporation residue studies of systems as massive as ${ }^{124} \mathrm{Sn}+{ }^{94} \mathrm{Zn} \rightarrow{ }^{218} \mathrm{Th}$, for bombarding energies at the Coulomb barrier, show strongly enhanced fusion cross sections, ${ }^{10}$ as well as the production of element 107 in the reaction ${ }^{54} \mathrm{Cr}+{ }^{209} \mathrm{Bi} \rightarrow{ }^{263} 107$, at the rate of a few events. ${ }^{11}$

For collisions of slow heavy ions, where the composite mass is less than that of a superheavy ion, it is generally accepted that a quasimolecule of this type is formed during a collision. Theoretical calculations of $\mathrm{Kr}$ and $\mathrm{Ca}$ induced collisions using the time dependent Hartree-Fock (TDHF) approximation show the formation of a fused system at energies near the Coulomb barrier. ${ }^{12-24}$ In such studies the final TDHF states yield extended matter distributions characterized by two centers, and having shell structure similar to that of the projectile and target fragments rather than those corresponding to the compound nucleus. Experimental probes of such structures have focused on the study of the atomic processes associated with the formation and deexcitation of the heavy quasiatom. ${ }^{15}$ Detailed information on the structure of the quasiatomic wave functions has been obtained from delta electron spectroscopy ${ }^{16}$ and also from the corresponding molecular orbital $x$ rays ${ }^{17}$ in a variety of systems. However, the complexities of the collision dynamics, collision broadening, nucleus-nucleus bremsstrahlung and inelastic photonuclear processes have lead to difficulties in obtaining detailed information concerning the nuclear dynamics. ${ }^{18}$

Besides these processes, the rapid time varying high $Z$ Coulomb field gives rise to both induced and spontaneous electron positron pair production from the neutral vacuum. These nonperturbative mechanisms have been extensively studied by Greiner and coworkers, ${ }^{19-24}$ who suggest that they form clear signatures for the nuclear dynamics. For systems having a combined charge of about 173, the 
inner shell electronic level structure is dominated by the relativistic dynamics, with the accompanying spontaneous positron emission and the formation of a charged vacuum. The treatment of the strong electromagnetic forces usually proceeds with the assumption of adiabatic motion in a quasimolecular picture. ${ }^{25}$ Magnetic and retardation effects have been investigated and for the most part can be neglected. ${ }^{16}$ The spontaneous positron emission usually corresponds to the decay of the $1 S_{\sigma}$ resonance from the negative energy continuum. The conditions for establishing the supercritical field occur only whenever the nuclear separation distance is less than a critical distance $R_{0}$, which usually happens for times of $10^{-21} \mathrm{sec}$ or less. This is in contrast to the resonance decay time which is usually greater than $10^{-19} \mathrm{sec}$. Thus the positron spectra from supercritical collisions are not expected to differ substantially from subcritical collisions. ${ }^{26}$

However, a clear signal for the supercritical emission occurs whenever the nuclear collision time is long compared to $10^{-21} \mathrm{sec}$, thus leading to a characteristic enhancement and structure in the spectrum. A number of measurements of the positron spectrum using $\mathrm{Pb}$ and $\mathrm{U}$ probes, have been carried out, including total and differential cross sections. The bombarding energy was varied over a range of energies near the Coulomb barrier. The $\mathrm{Pb}+\mathbf{P b}$ as well as the $\mathbf{P b}+\mathrm{U}$ systems are undercritical as quasimolecules under all conditions and exhibit only induced and direct positron emission. The $U+U$ system can in principle exhibit overcritical spontaneous pair creation. Recent experimental measurements by Greenberg et al. ${ }^{27}$ of $\mathrm{U}+\mathrm{U}$ collisions at $5.9 \mathrm{MeV}$ per particle have observed structure in the emitted positron spectrum. They suggest a separation of the quasimolecular positrons from the nuclear positron processes, and they see that the peaks in the spectra can be localized to heavy ion trajectories at specific angles.

In the present work we investigate the dynamics of the $\mathrm{U}+\mathrm{U}$ collision at energies near the Coulomb barrier using the TDHF approximation. Our basic motivation is to calculate the formation of a long-lived molecular complex and estimate the cross section for formation and angular focusing of the nuclear trajectories. Our calculations employ the finite-range Skyrme II and Skyrme III interactions together with a constant gap BCS pairing model. ${ }^{28}$ The TDHF approximation contains both the single particle (shell) as well as the collective degrees of freedom in a semiclassical picture, and allows for the direct calculation of the time evolution of heavy ion collisions. ${ }^{29}$ In this work we use the axial clutching model, which is approximately correct for near head-on collisions, ${ }^{29}$ and no spinorbit interaction. The omission of the spin-orbit force from the Hamiltonian is the most serious drawback of the present study, since it leads to incorrect shell structure for the ${ }^{238} \mathrm{U}$ nucleus. ${ }^{30}$

The usual method of treating heavy ion collisions in the TDHF approximation requires the preparation of an initial static Hartree-Fock (HF) wave function for each nucleus which is rotationally invariant and thereby simplifies the collisional dynamics. However, in our case we consider the propagation of intrinsically deformed initial states corresponding to the ground and excited states of ${ }^{238} \mathrm{U}$. Thus, besides the usual questions of propagation and interpretation of observables within the TDHF formalism, there are the added questions and complexities of collisions between intrinsically deformed nuclei. We consider only the propagation of stable solutions of the static HF equations corresponding to various minima in the collective potential energy surface of ${ }^{238} \mathrm{U}$. Previous studies of fission dynamics ${ }^{28}$ have investigated the collective potential energy surface of ${ }^{238} U$ in the static HF approximation. Our results for ${ }^{238} U$ are qualitatively similar and show several stable minima as a function of quadrupole deformation corresponding to isomeric states in the ${ }^{238} \mathrm{U}$ system. We treat the axially symmetric collisions of ${ }^{238} \mathrm{U}+{ }^{238} \mathrm{U}$, oriented along the $z$ axis, as a function of the bombarding energy. For energies corresponding to the measurements of Greenberg et .al., ${ }^{27} E_{\text {lab }}=1404 \mathrm{MeV}$, the collision of two uranium nuclei in their ground states have very little overlap at their distance of closest approach, and practically no nuclear time delay. However, whenever the U nuclei are highly deformed, there can be considerable nuclear interaction and time delays on the order of $5 \times 10^{-21} \mathrm{sec}$. The maximum calculated time delay is limited by a ternary fission breakup mode. The asymptotic initial states of the $U+U$ scattering are $J=0$ spherical ground states. This can be approximated in the TDHF model by doing a spherical HF-BCS approximation to the ground state of $U$, and using this initial state for the TDHF calculation. Again, this shows little nuclear delay at $1404 \mathrm{MeV}$. During the approach in the Coulomb field, the $J=0$ state of the deformed ground state band is Coulomb polarized and has an amplitude for being vibrationally Coulomb excited to the second minimum. Since we start the collision calculation almost at the touching point, in any case, we bypass the excitation process by assuming configurations at given orientations and deformations, so as to be able to work with a simple axial TDHF representation. The Coulomb excitation probabilities will multiply our cross sections; these probabilities are not computed here since our aim, in this work, is to study the amount of nuclear delay once a favorable configuration is reached. A favorable configuration minimizes the repulsive Coulomb force while maximizing the attractive nuclear force. Jensen and Wong ${ }^{31}$ have shown in the classical limit for grazing trajectories that the dynamical Coulomb polarization mainly effects the quadrupole and octupole moments of the ions. The major effects arise as a quadrupole polarization. In view of the exploratory nature of the present work, the octupole deformations were neglected. It is doubtful that their inclusion would substantially alter the present results. Apart from quadrupole polarization effects, the Coulomb force is $\simeq e^{2} Z_{1} Z_{2} / R_{s}^{2}$, where $R_{s}$ is the separation between centers when the nuclear densities overlap by $\simeq 50 \%$. The nuclear force is the attractive surface bond created when the two clusters touch. It is of the order or $20 \mathrm{MeV} / \mathrm{fm}$. As we shall see, even the most favorable configurations yield only a modest nuclear delay; a discussion of how this could change when spin-orbit effects are included, will be presented.

In the present study we include BCS pairing in computing the static Hartree-Fock solutions but we freeze the pairing amplitudes during the time evolution. Reference 32 has studied the time dependence of the BCS part of the TDHF dynamics. They find that the pairing energy varies between $-25 \mathrm{MeV}$ and $-10 \mathrm{MeV}$ for deeply inelastic symmetric uranium collisions. However, they find for 
TABLE I. Parameters of the modified Skyrme interaction.

\begin{tabular}{lcc}
\hline \hline Skyrme force & II & III \\
\hline$t_{0}\left(\mathrm{MeV} \mathrm{fm}^{3}\right)$ & -104.49 & -334.2930 \\
$x_{0}$ & 4.01 & 1.7458 \\
$t_{1}\left(\mathrm{MeV} \mathrm{fm}^{5}\right)$ & 585.60 & 395.0 \\
$t_{2}\left(\mathrm{MeV} \mathrm{fm}^{5}\right)$ & -27.10 & -95.0 \\
$t_{3}(\mathrm{MeV} \mathrm{fm}$ & 6 & 14000.0 \\
$a(\mathrm{fm})$ & 9331.00 & 0.45979 \\
$V_{l}(\mathrm{MeV})$ & 0.45979 & -355.864 \\
$V_{u}(\mathrm{MeV})$ & -444.85 & -619.735 \\
\hline \hline
\end{tabular}

grazing collisions that the variation is less than $3 \mathrm{MeV}$. In our work we have simplified the dynamical calculations by omitting this part of the interaction. Section II presents our results for the static properties of ${ }^{238} \mathrm{U}$ in the quadrupole constrained HF calculations used to initialize the TDHF dynamics. Section III details the time evolution of the various scattering configurations, while Sec. IV discusses the results.

Detailed discussions of the TDHF method are available in the literature $e^{33-49}$ and will not be presented here. We need only recall that the extension to include a constant gap BCS pairing interaction is straightforward. ${ }^{33-35}$ Using a time-dependent variational principle ${ }^{38}$ one obtains a formulation in terms of the pairing amplitudes $U_{\lambda}, V_{\lambda}$ which in the present work are time independent constants. Two versions of the modified Skyrme force $\mathrm{e}^{41-43}$ as given in Table $I$ are used.

\section{INITIAL SOLUTIONS}

The initial TDHF wave function is taken at two nonoverlapping static HF solutions whose centers translate toward one another along appropriate Coulomb trajectories. For spherically symmetric static solutions, the initial state is specified in terms of the entrance channel angular momentum (or equivalently the impact parameters) and the bombarding energy. However, for the collision of two deformed fragments, the relative orientation of the two fragments introduces five additional degrees of freedom (Euler angles) which are needed to specify the initial state. As discussed in Secs. I and IV, we restrict ourselves to axially symmetric static solutions oriented along the collision axis to obtain maximum overlap of the two fragments. Thus, we implicitly assume that those trajectories having the largest overlap will display the largest loss of kinetic energy and the longest time delay.

The static HF solutions $\phi_{\lambda}$ are obtained as a special class of solutions to the TDHF equations which have only an exponential time dependence of the form

$$
\psi_{\lambda}(r, t)=\phi_{\lambda}(r) \exp \left(-i \epsilon_{\lambda} t\right) .
$$

In the static limit the time dependent single particle Eqs. become a set of nonlinear equations for the static wave functions $\phi_{\lambda}$, the real single particle energies $\epsilon_{\lambda}$, and the expansion coefficients $v_{\lambda}$, namely

$$
\begin{aligned}
& h \phi_{\lambda}=\epsilon_{\lambda} \phi_{\lambda}, \\
& \operatorname{Im}\left\{v_{\lambda}\right\}=0, \\
& u_{\lambda}=\left(1-v_{\lambda}^{2}\right)^{1 / 2}, \\
& v_{\lambda}=\left\{\frac{1}{2}\left(1-\left(\epsilon_{\lambda}-\mu\right) /\left[\left(\epsilon_{\lambda}-\mu\right)^{2}+\Delta^{2}\right]^{1 / 2}\right\}^{1 / 2} .\right.
\end{aligned}
$$

For the static HF initial states, these equations are solved self-consistently using appropriate iteration methods. The constant gap pairing theory is equivalent to a corresponding Landau-Zener level crossing problem, ${ }^{50}$ and since the time evolution supresses level crossings, ${ }^{51}$ we include the BCS pairing only to determine the initial static HF wave functions. This means that the occupation numbers $\eta_{\lambda}$ are kept constant during the time evolution. However, this has the effect of supressing the dissipation that comes from the pairing field. Collisions in the lab frame between a projectile, mass number $A_{1}$, and a target, mass number $A_{2}$, with corresponding static HF solutions $\phi_{\lambda 1}, \phi_{\lambda 2}$, are initiated by specifying the initial state as

$$
\begin{aligned}
\psi_{\lambda}(r, t=0) & =\exp (i k \cdot r) \phi_{\lambda}\left(r-R_{1}\right), \quad \lambda=\{\lambda 1\}, \\
& =\phi_{\lambda}\left(r-R_{2}\right), \quad \lambda=\{\lambda 2\},
\end{aligned}
$$

where $R_{1}$ and $R_{2}$ are the initial coordinates of the centers of $A_{1}$ and $A_{2}$, respectively, and for a bombarding energy $E_{\text {lab }}$,

$$
k=\left(2 m E_{\mathrm{lab}} / A_{1}\right)^{1 / 2} .
$$

In constructing static HF solutions for uranium, we consider a family of axially symmetric solutions as a function of the quadrupole deformation $q$. These solutions are obtained by adding an external quadrupole field to the Hamiltonian so that the energy functional becomes

$$
\begin{aligned}
& E(q)=E_{\text {field }}+E_{\text {pair }}+c\left(\left\langle Q_{20}\right\rangle-q\right)^{2}, \\
& \partial E(q) / \partial c=0 .
\end{aligned}
$$

The last equation ensures that the total energy of the system is an extremum for the deformation $q$. As a function of the deformation, the interplay between the shape degrees of freedom and the pairing modes plays a central role in determining the minimum energy.

We solve both the static HF and the TDHF equations directly in coordinate space using the finite difference techniques reported in Ref. 29, and for the static HF equations, the imaginary time method in Ref. 52. These equations are obtained by discretizing in space and time the energy functional. The Hamiltonian density is assumed to be symmetric about the axis joining the centers of the two ions, and is only a function of two space coordinates, $z$ and $r$, respectively, the displacement parallel to the symmetry axis, and the perpendicular displacement. The spacetime discretization results in the specification of wave functions and operators on the mesh points $r_{m}, z_{n}$ at a time $t_{j}$, as

$$
\psi_{\lambda}(m, n, j)=\psi_{\lambda}\left(r_{m}, z_{n}, t_{j}\right)
$$

with

$$
\begin{aligned}
& r_{m}=\left(m-\frac{1}{2}\right) \delta, \quad m=1,2, \ldots, N_{r}, \\
& z_{n}=(n-1) \delta, \quad n=-N_{z},-N_{z}+1, \ldots, N_{z}-1, N_{z}, \\
& t_{j}=j \Delta t, \quad j=0,1,2, \ldots, .
\end{aligned}
$$

The full description of these procedures are given in Refs. 29 and 52. Detailed analysis of the application of these methods to the study of the ${ }^{236} \mathrm{U}$ system using the Skyrme II force were given in Ref. 28. The relevant points are summarized below. For a mesh spacing $\delta=1.0 \mathrm{fm}$, the 
TABLE II. Comparison of the static isomer state properties of ${ }^{238} \mathrm{U}$ for two different numerical grids, using Skyrme III with $\Delta=0.71 \mathrm{MeV}$.

\begin{tabular}{lcc}
\hline \multicolumn{1}{c}{$\delta(\mathrm{fm})$} & 1.0 & 0.8 \\
\hline$\langle T\rangle(\mathrm{MeV})$ & +4433.4 & +3998.9 \\
$U_{0}(\mathrm{MeV})$ & -6479.2 & -6480.3 \\
$E_{0}(\mathrm{MeV})$ & -2045.8 & -2481.4 \\
$E_{\text {pair }}(\mathrm{MeV})$ & -22.2 & -21.9 \\
$\left\langle Q_{20}\right\rangle\left(\mathrm{fm}^{2}\right)$ & +3865.9 & +3671.8 \\
$R_{0}(\mathrm{fm})$ & 6.738 & 6.367 \\
\hline \hline
\end{tabular}

principle truncation error is due to the approximate treatment of the kinetic energy operator and results in a six percent error in the total HF energy. The shape of the deformation energy curve is stable with respect to both decreases and increases in $\delta$, with negligible changes in the position and relative depth of the minima. Also, no significant truncation errors occur for the surface energy, 43 supporting the claim that the truncation occurs as a volume contribution to the kinetic energy, with an overall error in the deformation energy curve of about $10 \%$.

Additional errors are introduced by the truncation of the single particle space needed to solve the BCS pairing equations. In our calculation we include single particle states having energies up to about $+10 \mathrm{MeV}$, which yield pairing matrix elements $g=0.13$, and $0.17 \mathrm{MeV}$ corresponding to gaps of $\delta=0.71$ and $2.0 \mathrm{MeV}$. Realistic residual matrix elements range between 0.0 and $0.4 \mathrm{MeV}$ in the uranium region depending on the basis and the effective interaction employed. ${ }^{53,54}$

In Table II we compare the bulk properties of the static HF solutions for ${ }^{238} U$ using the Skyrme II and the Skyrme III forces with a pairing gap of $\Delta=0.71 \mathrm{MeV}$. Two different values of the mesh spacing, $\delta=1.0 \mathrm{fm}$ and $\delta=0.80$ fm are compared with $N_{r} \times N_{z}=24 \times 40$ points. The static properties of uranium are approximately independent of the force, the largest difference occurring for the higher moments of the mass density and indicating that the major difference between the two interactions occurs in the surface. The binding energy difference between the two meshes seems to arise from the difference in the kinetic energy terms, and is less than $10 \%$ as reported in the earlier work. The similarities of the deformation energy curves for both of these forces was reported for the induced fission of ${ }^{236} \mathrm{U}$.

The deformation energy curve for the Skyrme III interaction is shown in Fig. 1 for two values of the pairing gap, $\Delta=0.71 \mathrm{MeV}$ and $\Delta=2.0 \mathrm{MeV}$, as a function of the mass quadrupole moment. Our results are essentially the same as those obtained in Ref. 43. The deformation scaling parameter $q_{0}$ is defined as

$$
\begin{aligned}
q_{0} & =\left(\frac{5}{16} \pi\right)^{1 / 2} R^{2} A \\
& =3879.0 \mathrm{fm}^{2},
\end{aligned}
$$

where $R=7.189 \mathrm{fm}$ is the approximate strong interaction radius of uranium. The solutions in Fig. 1 are generated using an iteration procedure in which the solutions at $q$ are used as an initial guess for the solutions at $q+\delta q$. Our initial wave function guesses for the static HF procedure are

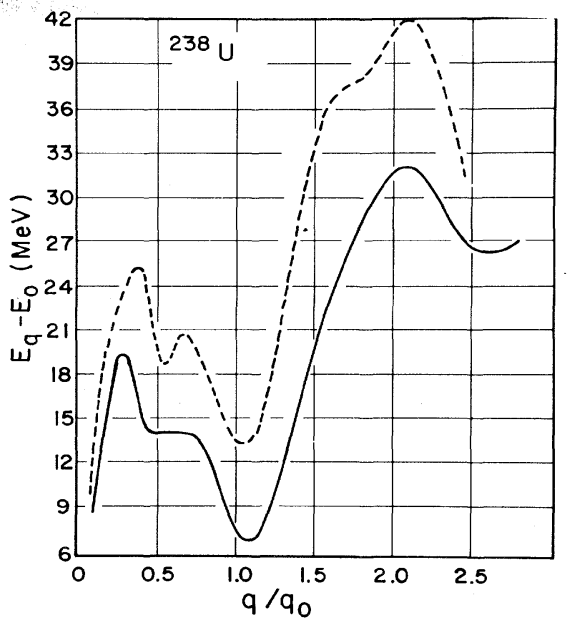

FIG. 1. The static HF energy of deformation $E_{q}-E_{0}$, as a function of the quadrupole moment $q$ calculated with the Skyrme III force and with $\Delta=0.71 \mathrm{MeV}$ (solid line), and with $\Delta=2.0 \mathrm{MeV}$ (dashed line); $q_{0}$ is defined in the text.

obtained from deformed harmonic oscillator states. The restriction to axial symmetry for large deformations is a serious approximation and leads to unphysical enhancements in the fission barriers. ${ }^{55}$ However, we are interested in the solution near $q=1.10 q_{0}$, which is a stable solution of the unconstrained $(c=0)$ static HF equations. The lowest energy solution (not shown in Fig. 1) is more than a factor of 25 less prolate than the intrinsic empirical deformation, a result of the suppression of the spin-orbit part of the interaction. Note that the occupation numbers $\eta_{\lambda}$ are constant in time. These are shown for neutrons in Fig. 2 as a function of the single particle energies $\epsilon_{\lambda}$ for the isomeric state having $q=1.10 q_{0}$, and calculated using the Skyrme III force with $\delta=0.71 \mathrm{MeV}$.

\section{TIME EVOLUTION}

We performed head-on $(l=0)$ symmetric collisions of two ground state uranium nuclei. At the bombarding en-

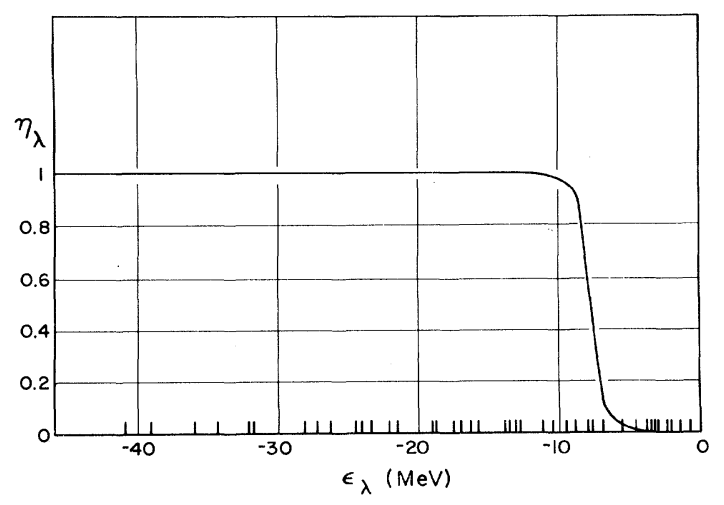

FIG. 2. The neutron occupation numbers as a function of the HF single particle energy for the isomer state with $q / q_{0}=1.10$, calculated with the Skyrme III force and $\Delta=0.71 \mathrm{MeV}$. 
TABLE III. Comparison of the static ground and isomer state properties of ${ }^{238} U$ for the interactions studied.

\begin{tabular}{lcccc}
\hline \hline & II & $\begin{array}{c}\text { Skyrme force } \\
\text { II }\end{array}$ & III & III \\
& & $\Delta(\mathrm{MeV})$ & & \\
& 0.71 & 2.0 & 0.71 & 2.0 \\
\hline Ground state & & & & \\
$E_{0}(\mathrm{MeV})$ & -2107.8 & -2154.0 & -2050.0 & -2106.1 \\
$\left\langle Q_{20}\right\rangle\left(\mathrm{fm}^{2}\right)$ & -37.8 & +213.0 & +343.5 & +347.3 \\
$R_{0}(\mathrm{fm})$ & 5.690 & 5.694 & 5.753 & 5.762 \\
$E_{\text {pair }}(\mathrm{MeV})$ & -15.4 & -93.4 & -21.1 & -118.0 \\
Isomer state & & & & \\
$E_{0}(\mathrm{MeV})$ & -2099.1 & -2146.3 & -2045.8 & -2106.9 \\
$\left\langle Q_{20}\right\rangle\left(\mathrm{fm}{ }^{2}\right)$ & +2171.6 & +2019.8 & +3865.9 & +3879.1 \\
$R_{0}(\mathrm{fm})$ & 6.114 & 6.077 & 6.738 & 6.748 \\
$E_{\text {pair }}(\mathrm{MeV})$ & -15.6 & -101.2 & -22.2 & -124.2 \\
\hline \hline
\end{tabular}

ergy near the Greenberg positron measurements $E_{\text {lab }}$ $=1404 \mathrm{MeV}$, the TDHF trajectories correspond closely to classical Coulomb trajectories of two point charges, with no appreciable time delay. These cases are not discussed further here. There are other possibilities to provide a time delay at this energy. These involve the collisions of strongly deformed configurations. For example, in collisions of light systems, ${ }^{56,57}$ long-lived molecular configurations are studied by considering the TDHF collisions of ground and isomeric states of ${ }^{12} \mathrm{C}$. These collisions display many of the properties associated with the quasimolecular phenomena, and, in particular, the long interaction times characteristic of resonant structures. We note also that molecular structures were found in TDHF calculations of $\mathrm{Kr}+\mathrm{La}$ collisions with lifetimes of more than $10^{-20}$ sec. $^{12,3}$ In the uranium system the isometric states are well established and could act as doorways to molecular structures in the $U+U$ collision. Accordingly we have investigated the collision of aligned isomer states of uranium $\left(U^{*}\right)$ for a range of bombarding energies, for both Skyrme II and Skyrme III interactions (see Table III). The collision time for the $U^{*}+U^{*}$ head-on collision is

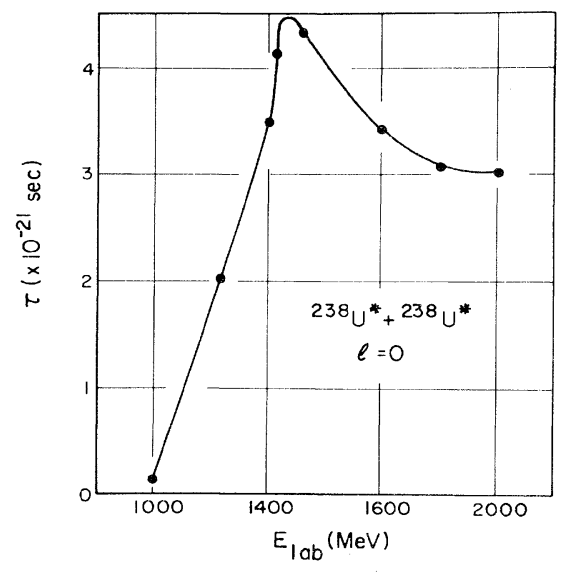

FIG. 3. The time delay $\tau$ as a function of the laboratory bombarding energy for head-on symmetric collisions of two isomeric uranium, using the Skyrme II force. shown in Fig. 3 as a function of the bombarding energy. The maximum time for which the system remains coalesced is less than $1200 \mathrm{fm} / c$, e.g., about $4.0 \times 10^{-21}$ sec. This is obtained with the Skyrme III force and a pairing gap of $0.71 \mathrm{MeV}$. Figure 4 shows the delay time for the $\mathrm{Kr}+\mathrm{Pb}$, for comparison. A much longer delay time is observed. We note that there is a difference in the evolution between the two forces, as can be seen by comparing the final state matter distributions in Figs. 5 for $E_{\text {lab }}=1404 \mathrm{MeV}$. Figures 5 show the equidensity contours in the collision plane at various times during the collision, for Skyrme II [Fig. 5(a)] and for Skyrme III [Fig. 5(b)]. The evolution with force II leads to a two-body final state, as do the trajectories in Figs. 3 and 4. However, force III at this energy, generates a much longer time evolution and the system eventually comes apart into three separate fragments [Fig. 5(b)]. Ternary fission processes have not been extensively studied experimentally and it is not known how important they are in the uranium collision. These are symmetric collisions between identical nuclei, and the final state must also have this symmetry. The mass of each of the smaller fragments in Fig. 5(b) is about $100 \mathrm{u}$, so that the central fragment of the final state has a mass comparable to that of uranium. Both the time

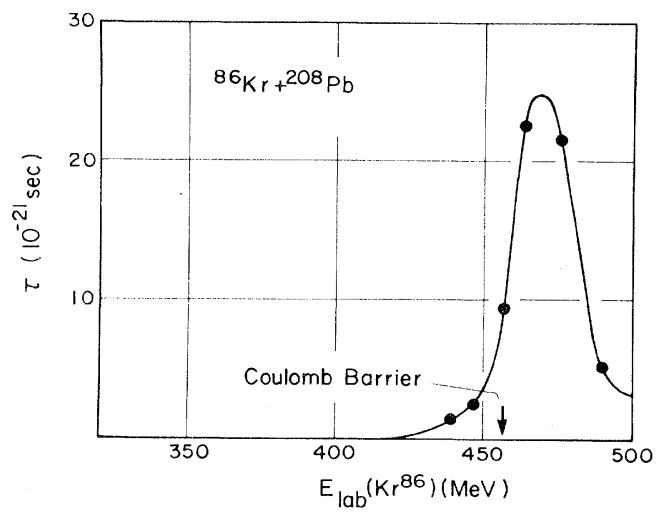

FIG. 4. Same as Fig. 3 for the collision of ${ }^{86} \mathrm{Kr}+{ }^{208} \mathrm{~Pb}$. 

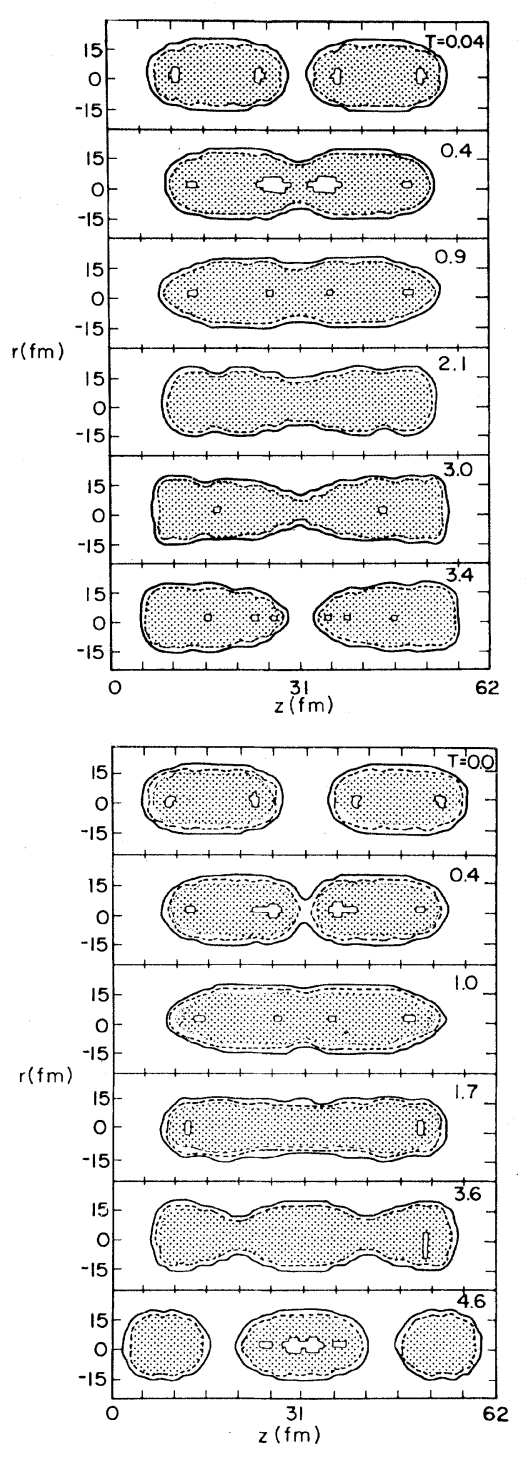

FIG. 5. (a) Density contours for the $U+U$ isomers head-on collision at $1404 \mathrm{MeV}$ and for Skyrme II. The solid line represents approximately the $10 \%$ density contour, while the dashed line stands for the approximate half-density contour. Binary fission is observed after only a modest delay time. The times indicated are in units of $10^{-21} \mathrm{sec}$. (b) Same as (a) but for Skyrme III. Ternary fission is observed instead of binary fission. Note that the times for the first plot in (a) and (b) are different.

scale and energy loss of these reactions is consistent with TDHF descriptions of deep inelastic processes in this mass region. ${ }^{37}$

As a comparison we have also studied the ground state collisions of ${ }^{86} \mathrm{Kr}+{ }^{208} \mathrm{~Pb}$ at energies near the Coulomb barrier. The collision time is given in Fig. 4 as a function of the lab energy. Again we are considering only $l=0$ trajectories. The long time delay which occurs $20 \mathrm{MeV}$ above the Coulomb barrier $\left(E_{\text {lab }}=464 \mathrm{MeV}\right)$ shows a structure characteristic of the molecular configurations re-

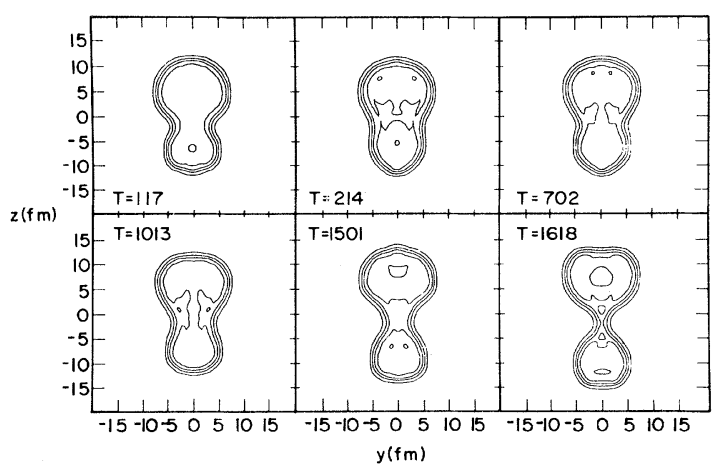

FIG. 6. Equidensity contours in the reaction plane for the collision of ${ }^{85} \mathrm{Kr}+{ }^{208} \mathrm{~Pb}$.

ported in Ref. 13. The density contours in the collision plane are given as a function of time in Fig. 6 for this reaction. Investigation of other systems indicate that the occurrence of these structures are linked to details of the particular shell structure and the behavior of the interaction in the nuclear surface. We return to this matter in the discussion.

For non-head-on collisions, the axial symmetry condition leads to further approximations to the nuclear dynamics. Nevertheless, collisions can be carried with a reasonable degree of certainty for the energy loss and final scattering angle of the outgoing fragments, as long as the bombarding energy is not too large. The final kinetic energies and scattering angles for the $E_{\text {lab }}=1404 \mathrm{MeV}$ $\mathbf{U}^{*}+\mathbf{U}^{*}$ collision are shown in Fig. 7 as a function of the angular momentum $l$. The extended aligned prolate configuration of the isomeric uranium yields a forward angular focusing of the final fragments as well as a sharp

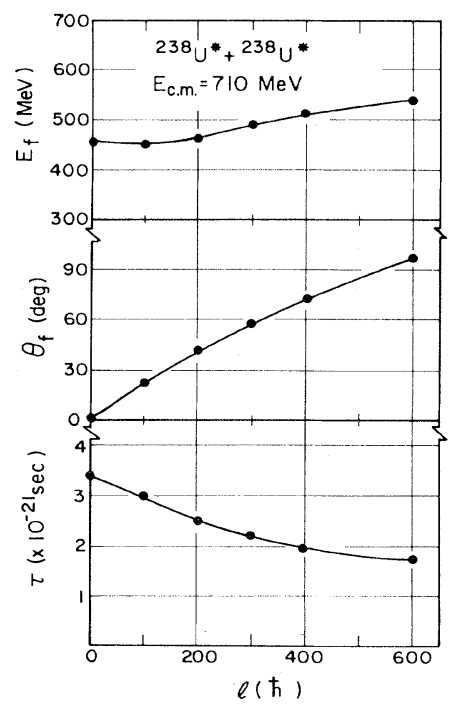

FIG. 7. Outgoing fragment kinetic energies and scattering angles in the c.m. frame and time delays as a function of the entrance channel angular momentum for the symmetric collision of two uranium isomers. 


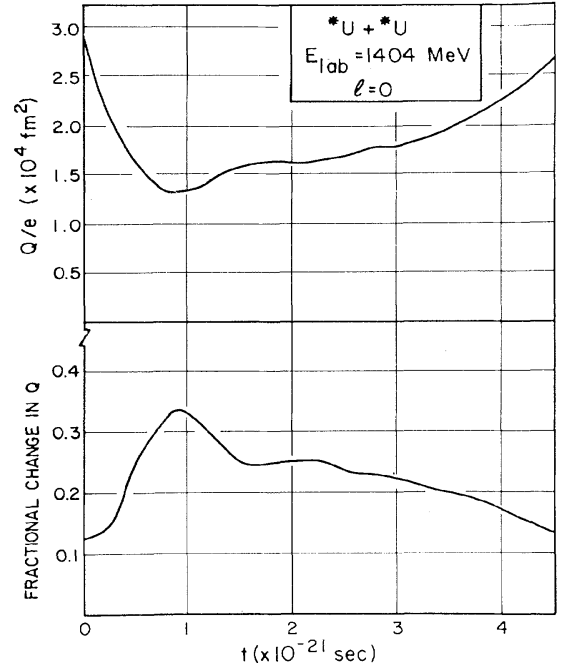

FIG. 8. The charge quadrupole moment for the isomeric uranium system as a function time, together with the fractional deviation in $q$ for two point nuclei moving on equivalent trajectories.

structure in the nuclear time delay function.

Calculations of the positron emission spectrum have generally been carried out assuming a time dependent monopole charge distribution for the nuclear field. ${ }^{24}$ Estimate $^{22}$ have indicated that nuclear quadrupole contributions to the electric field can significantly broaden the positron distribution. In Fig. 8 the total nuclear charge quadrupole moment is given as a function of time, together with the fractional deviation of the quadrupole moment from that obtained with point nuclei moving on the same trajectories. One sees that the internal excitations of the uranium lead to modifications of about $30 \%$ at most. The large moment of the coalesced system arises in part from the large intrinsic moment of the uranium isomers.

\section{DISCUSSION AND SUMMARY}

We have found that it is not possible to obtain an appreciable nuclear time delay in the reaction $U+U$ at $E_{\text {lab }}=1404 \mathrm{MeV}$ unless we assume that one or both uranium nuclei are excited into configurations with large deformations, the isomeric states. The probability that uranium will undergo this transition is governed by Coulomb excitation processes. In the most favorable circumstance, the isomer excitation probability is of the order of $10^{-3}$ to $10^{-2}$. The positron emission data is not inconsistent with such small excitation probabilities, and reliable calculations of this transition probability are needed before more definite conclusions are drawn. However it is known that nuclear delay times of about $10^{-20}$ to $10^{-19} \mathrm{sec}$ are required in order to explain the observed width of the positron distributions, whereas the maximum time delay in our calculations is less than $4.0 \times 10^{-21} \mathrm{sec}$, about an order of magnitude too small. We shall now discuss in some detail the possible reasons for this result.

First, long time delays at bombarding energies near the
Coulomb barrier are indeed possible, as illustrated in the ${ }^{86} \mathrm{Kr}+{ }^{208} \mathrm{~Pb}$ calculation described earlier. The Coulomb repulsion for $\mathrm{Kr}+\mathrm{Pb}$ is much smaller than for $\mathrm{U}+\mathrm{U}$. One can develop a quantitative comparison of the effects of the Coulomb repulsion and the nuclear attraction by writing the following estimates. The Coulomb force between two charges $Z_{1}$ and $Z_{2}$ whose centers are separated by $R_{12}$ is

$$
F_{c}=e^{2} Z_{1} Z_{2} / R_{12}^{2} \text {. }
$$

For $\mathrm{Kr}+\mathrm{Pb}$ at the strong interaction radius $R_{12}=15.4$ $\mathrm{fm}, F_{c}$ is about $19.4 \mathrm{MeV} / \mathrm{fm}$. In order for the system to remain together this force must be less than the attractive nuclear surface interaction. This is estimated by noting that the gain in potential energy in letting the surfaces of the two nuclei come together is about $35 \mathrm{MeV}$, which occurs over a distance of about $1.5 \mathrm{fm}$. Thus $F_{s}$, the nuclear surface force, is about $23 \mathrm{MeV}$. In the $\mathrm{Kr}+\mathrm{Pb}$ case, the nuclear attraction dominates the Coulomb repulsion and we see a long-lived resonance near the top of the Coulomb barrier. We have examined other cases near the Coulomb barrier, in particular, ${ }^{142} \mathrm{Nd}+{ }^{142} \mathrm{Nd}(824 \mathrm{MeV})$, ${ }^{154} \mathrm{Nd}+{ }^{154} \mathrm{Nd}(738-781 \mathrm{MeV}),{ }^{154} \mathrm{Sm}+{ }^{154} \mathrm{Sm} \quad(755-893$ $\mathrm{MeV}),{ }^{166} \mathrm{Er}+{ }^{166} \mathrm{Er} \quad(880-974 \mathrm{MeV})$, and ${ }^{186} \mathrm{~W}+{ }^{186} \mathrm{~W}$ $(1042-1135 \mathrm{MeV})$. This represents a range of Coulomb forces, at the strong absorption radius, of $22 \mathrm{MeV} / \mathrm{fm}$ to $29 \mathrm{MeV} / \mathrm{fm}$. These are all stronger than the Coulomb force of $\mathrm{Kr}+\mathrm{Pb}$, and in all of these cases no barrier resonance is found. This suggests that the limit for the formation of the resonance is a Coulomb force of about 20 $\mathrm{MeV} / \mathrm{fm}$. The actual value will vary from nucleus to nucleus because shell structure will affect the nuclear attraction. In particular, one can expect an additional 3-4 MeV of attraction in the most favorable cases. The present calculation with the uranium isomer does not have the correct shell structure since it lacks the spin-orbit force, and does not give a long lived resonance at energies near the Coulomb barrier. These isomeric states are strongly deformed and yield a Coulomb force $F_{c}$ at the strong absorption radius, $F_{c} \simeq 22 \mathrm{MeV} / \mathrm{fm}$. In order for this system to form a long lived molecule, we would need an additional surface attraction force of some $2-3 \mathrm{MeV} / \mathrm{fm}$, which would have to come from the neglected spin orbit interaction.

The occurrence of three-body breakup in our calculation is another reason for the short lifetime of the compound nucleus. It is known that shell effects will strongly affect the barriers for such decay channels. Since we do not have the correct shell and spin-orbit effects, it is possible that our three-body decay mode is spurious. The absence of such a mode could increase our observed lifetime considerably, so that our conclusion regarding the $U+U$ nuclear delay time is once more made uncertain by a substantial factor, possibly as much as a factor of 10 .

We note that the excitation energy in the compound system can be quite small at energies near the top of the Coulomb barrier. This is due in part to the formation of a neck during the collision, and is usually accompanied by strong octupole deformations of one or both fragments. This effect can be enhanced whenever the fragments have a high octupole polarizability, as is the case with the uranium ground and isomeric states, and several isotopes in other regions of the Periodic Table, such as in the $\mathrm{Ni}$ 
region. We also note that the lower the excitation energy after neck formation the more important is the shell structure and the more favorable are the chances to increase (or decrease) the surface attraction with the help of shell fluctuations. Calculations of TDHF reactions including the spin-orbit force are currently underway and may lead to the long lived $U+U$ compound system needed to explain the positron emission data. As a preliminary step to the dynamical calculation one could perform static constrained HF calculations for the composite $U+U$ system. In particular, it should be possible to look for local mini$m a$ in the deformation energy surface, for shapes similar to the ones observed here. It is not even necessary that these be absolute minima in all the possible collective de- grees of freedom. It suffices that, for those degrees of freedom where we have only a saddle point, the corresponding inertia parameter $\left(m^{-1}\right)$ be small. This is the point at which TDHF needs to be done, since it is quite difficult to do reliable mass parameter calculations using constrained HF techniques.

\section{ACKNOWLEDGMENTS}

We acknowledge with pleasure stimulating conversations with Dr. P. Armbruster and Dr. A. Gobbi. This work was supported in part under U. S. Department of Energy Contract No. DE-AC02-76ER03074.
*Present address: National Superconducting Cyclotron Laboratory, Michigan State University, East Lansing, MI 48824.

${ }^{1}$ H. Meldner, Arkv. Fys. 36, 593 (1967).

${ }^{2}$ G. T. Seaborg, Phys. Scri. A10, 5 (1974).

${ }^{3}$ C. E. Bemis, Jr. and J. R. Nix, Comments Nucl. Part. Phys. $\underline{7}$, 5 (1977).

${ }^{4} \mathrm{G}$. Herrmann, Proceedings of the Fourth International Conference on Nuclei Far from Stability, Helsingør, Denmark, 1981 (unpublished), p. 772.

${ }^{5}$ U. Mosel and W. Greiner, Z. Phys. 222, 221 (1969).

6S. G. Nilsson, C. F. Tsang, A. Sobiczewski, Z. Szymanski, S. Wycech, C. Gustafson, I. L. Lamm, P. Moller, and B. Nilsson, Nucl. Phys. A131, 1 (1969).

${ }^{7}$ D. Vautherin, M. Veneroni, and D. M. Brink, Phys. Lett. $\underline{33 B}$, 381 (1970).

${ }^{8}$ W. Greiner, Universität Frankfurt, Theoretische Physik lecture notes, 1970 (unpublished).

${ }^{9}$ A. K. Dhar, B. S. Nilsson, K. T. R. Davies, and S. E. Koonin, Nucl. Phys. A364, 105 (1981).

${ }^{10}$ K. H. Schmidt et al., Nucl. Phys. A318, 253 (1979); C. C. Sahm et al., Gesellschaft für Schwerionenforschung Scientific Report ISSN0174-0814 1981 (unpublished), p. 5.

${ }^{11}$ G. Manzenberg et al., Z. Phys. A $\underline{300}, 107$ (1981); Gesellschaft für Schwerionenforschung Scientific report 1981 (unpublished), p. 67.

${ }^{12}$ K. T. R. Davies, K. R. Sandhya Devi, and M. R. Strayer, Phys. Rev. Lett. 44, 23 (1980).

${ }^{13}$ K. T. R. Davies, K. R. Sandhya Devi, and M. R. Strayer, Phys. Rev. C24, 2576 (1981).

${ }^{14}$ H. Stocker, R. Y. Cusson, H. J. Lustig, A. Gobbi, J. Hahn, J. A. Maruhn, W. Greiner, Z. Phys. A $\underline{306}, 235$ (1982).

15J. Greenberg, in Proceedings of the XIth International Conference on Electronic and Atomic Collisions, Kyoto, 1979, edited by K. Takayanagi and N. Oda (The Society for Atomic Collisional Research, Kyoto, 1979), p. 351.

16J. S. Greenberg, H. Bokemeyer, H. Emling, E. Grosse, D. Schwalm, and F. Bosch, Phys. Rev. Lett. 39, 1404 (1977).

17J. S. Greenberg, C. K. Davies, and P. Vincent, Phys. Rev. Lett. 33, 473 (1974); Phys. Rev. A18, 18 (1978).

${ }^{18}$ W. E. Meyerhof; T. K. Saylor, and R. Anholt, Phys. Rev. A12, 2641 (1975).

${ }^{19}$ W. Peiper and W. Greiner, Z. Phys. 218, 126 (1969).

${ }^{20}$ B. Muller, J. Rafelski, and W. Greiner, Z. Phys. 257, 183 (1972).

21J. Rafelski, B. Muller, and W. Greiner, Nucl. Phys. B68, 585 (1974).
22J. Reinhardt, B. Muller, W. Greiner, and G. Soff, Phys. Rev. Lett. 43, 1307 (1979).

23J. Reinhardt and W. Greiner, Rep. Prog. Phys. 40, 219 (1977).

2.4r Reinhardt, V. Oberacker, G. Soff, B. Muller, and W. Greiner, Phys. Rev. 78B, 183 (1978).

${ }^{25}$ B. Muller, Annu. Rev. Nucl. Sci. 26, 351 (1976).

26J. Reinhardt, U. Muller, B. Muller, and W. Greiner, Universität Frankfurt Theoretische Physik Report 63, 1981 (unpublished).

${ }^{27}$ H. Backe et al., Phys. Rev. Lett. 느, 1443 (1980); J. S. Greenberg, private communication.

28J. W. Negele, S. E. Koonin, P. Moller, J. R. Nix, and A. J. Sierk, Phys. Rev. C17, 1098 (1978).

${ }^{29}$ K. T. R. Davies and S. E. Koonin, Phys. Rev. C 233, 2042 (1981).

${ }^{30}$ H. C. Lee and R. Y. Cusson, Ann. Phys. (N.Y.) 72, 353 (1972).

${ }^{31}$ A. S. Jensen and C. Y. Wong, Nucl. Phys. A171, 1 (1971).

${ }^{32}$ R. Y. Cusson, J. A. Maruhn, and H. Stocker, Z. Phys. 294, 257 (1980).

33J. Blocki and H. Flocard, Nucl. Phys. A238, 45 (1976).

${ }^{34}$ R. Y. Cusson and H. Meldner, Phys. Rev. Lett. 42, 694 (1979).

35J. W. Negele, in Theoretical Methods in Medium Energy and Heavy Ion Physics, edited by $\mathrm{K}$. McVoy and W. A. Frieman (Plenum, New York, 1978).

${ }^{36}$ S. E. Koonin, Prog. Part. Nucl. Phys. 4, 283 (1980).

37J. W. Negele, Rev. Mod. Phys. (to be published).

${ }^{38}$ K. T. R. Davies, K. R. Sandhya Devi, S. E. Koonin, and M. R. Strayer, in Heavy Ion Science, edited by D. Allan Bromley (Plenum, New York, 1982).

${ }^{39}$ A. K. Kerman and S. E. Koonin, Ann. Phys. (N.Y.) 100,332 (1976).

${ }^{40}$ T. H. R. Skyrme, Philos. Mag. 1,1043 (1956).

${ }^{41}$ D. Vautherin and D. M. Brink, Phys. Rev. C $\underline{5}, 626$ (1972).

${ }^{42}$ P. Hoodbhoy and J. W. Negele, Nucl. Phys. A288, 23 (1977).

${ }^{43}$ R. Y. Cusson and J. A. Maruhn, Phys. Lett. B62, 134 (1976).

44J. A. Maruhn and R. Y. Cusson, Nucl. Phys. A270, 471 (1976).

${ }^{45}$ R. Y. Cusson, H. P. Trivedi, H. W. Meldner, and M. W. Weiss, Phys. Rev. C 14, 1615 (1976).

${ }^{46}$ P. Quentin and H. Flocard, Annu. Rev. Nucl. Part. Sci. 28 , 523 (1978).

${ }^{47}$ J. W. Negele and D. Vautherin, Phys. Rev. C $\underline{5}, 1472$ (1973).

48J. W. Negele, Phys. Rev. C 1 1260 (1970).

49J. W. Negele and D. Vautherin, Phys. Rev. C 11, 1031 (1975).

${ }^{50}$ L. Landau, Phys. Z. Sowjetunion $\underline{1}, 88$ (1932); $\underline{2}, 46$ (1932). 
51P. G. Reinhard, Nucl. Phys. A281, 221 (1977).

${ }^{52}$ K. T. R. Davies, H. Flocard, S. J. Krieger, and M. S. Weiss, Nucl. Phys. A342, 111 (1980).

${ }^{53}$ A. J. Sierk and R. J. Nix, Phys. Rev. C 21, 982 (1980).

${ }^{54}$ R. Y. Cusson, D. Kolb, and M. Harvey, Nucl. Phys. A215, 1 (1970).

${ }^{55}$ H. Flocard, P. Quentin, A. K. Kerman, and D. Vauterin, Nucl. Phys. A203, 433 (1973).
${ }^{56}$ D. Allan Bromley, Proceedings of the International Conference on Resonances in Heavy Ion Collisions, Bad Honnef, Federal Republic of Germany, 1981 (unpublished).

${ }^{57}$ M. R. Strayer, A. S. Umar, R. Y. Cusson, J. A. Maruhn, D. Allan Bromley, and W. Greiner, Proceedings of the Bad Honnef Conference on TDHF and Beyond, Bad Honnef, 1982; unpublished. 